\title{
Revolutionizing Spaceflight: A Study on Electric Propulsion and Air-Breathing MPDs
}

\author{
Ian Mckinney ${ }^{1}$ and John Murnan ${ }^{1 \#}$ \\ ${ }^{1}$ Etowah High School, Woodstock, GA, USA \\ \#Advisor
}

\section{ABSTRACT}

Modern liquid-fuel rocket propulsion harbors a number of great limitations. Among those is the weight of fuel, which makes up more than $90 \%$ of the mass of the SpaceX Falcon 9 (NASA, 2018). Electric propulsion has been used for decades as an alternative to liquid-fuel rockets due to low propellant requirements and high specific impulse. Although electric thrusters have strictly been used in non-atmospheric conditions, recent innovations attempt to expand its use to airspace. This quasi-experimental study focuses on the creation of an air-breathing magnetoplasmadynamic (MPD) thruster, with attempts being made to maximize the efficiency of the engine. Immense safety concerns prevented testing from occurring after the engine was built. However, the estimated performance of the built MPD is compared to a multitude of existing forms of electric propulsion, from Hall-effect thrusters to electrodynamic tethers. The concluding evidence suggests that air-breathing MPDs are not currently viable, high-power photon thrusters being of greater use in atmospheric conditions. Further research focusing on decreasing atmospheric breakdown voltage and increasing mirror reflectance of photon thrusters is suggested.

\section{Introduction \& Review of Literature}

April 12 $2^{\text {th }}$, 1961. 7:55 pm. The ejector seat launches Soviet cosmonaut Yuri Gagarin into open air seven kilometers above the ground. He looks down as his parachute releases, staring at the capsule falling towards the ground below him. For the first time in history, humanity successfully ventured into space. Since then, we have revolutionized spaceflight many times over. The Saturn V, first launched in 1967 for the Apollo lunar missions, remains today the most powerful rocket ever built. Power is not everything, however, as seen by the innovations made in the Columbia space shuttle and SpaceX's Falcon 9. All three of these crafts are incredibly different, but they are all extremely limited by one fatal aspect: fuel. Over $90 \%$ of the Falcon 9's 560- ton weight is solely fuel, making the thrust needed to escape the atmosphere exponentially more (National Aeronautics and Space Administration [NASA], 2018). This tradeoff between mass and thrust due to fuel is existent simply because no alternative has yet been successfully attempted for the first stage - the engine and fuel used from liftoff - of rockets. It is a completely different story, however, with the final stages of crafts. Electric propulsion systems have played an important role in space exploration and technology for decades, powering satellites and small probes by harnessing electrical energy in a variety of different fashions. Among the most common types are magnetoplasmadynamic (MPD) thrusters. In addition to this type of engine, 
alternative drives such as Hall-effect thrusters, solar sails, and electrodynamic tethers are also utilized for small spacecrafts and satellites, each having unique advantages over the others.

MPDs are a type of plasma thruster - a propulsion device that accelerates charged particles in addition to the loose electrons that are characteristic of plasma. There are multiple constructs of plasma engines, from pulsed plasma thrusters to helicon plasma thrusters, that all utilize this fundamental similarity. MPDs are further divided into two subgroups: self-field and applied-field. Both implement cathodes and anodes - electromagnetic devices that expel (anode) and attract (cathode) electric charge - to induce a magnetic field. Self-field thrusters use the field produced between a cathode rod in the center of the engine and a surrounding body acting as an anode. Applied-field thrusters, however, also use exterior magnetic coils to produce such an effect (Zheng et al., 2020). Both self-field and applied-

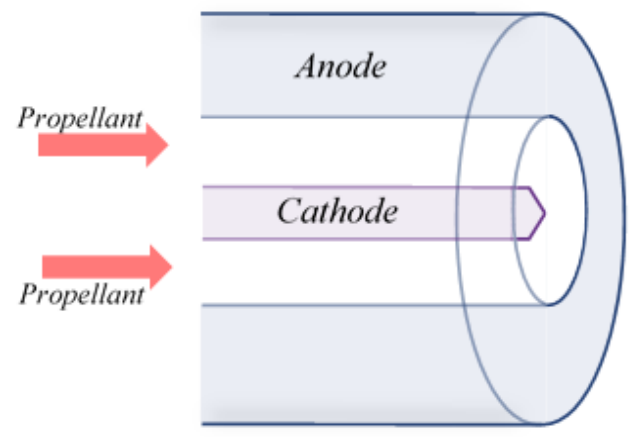

Figure 1. Diagram of a self-field MPD

field MPDs have two main structural mechanisms: an intake and an ionization chamber. The intake is usually a small hole in the engine's body, connecting the thruster to a tank of highly compressed gas. The gas travels inside the engine and reaches the ionization chamber, where electric arcs connecting the anode and cathode turn the gas into a plasma state. The plasma then interacts with the magnetic field produced by the engine, which pushes the gas out of the craft at incredibly high speeds. Argon, xenon, and helium gases are most used for MPDs largely due to their low breakdown voltages - the power needed to break down the gas into plasma. A low breakdown voltage allows for more electrical energy to be used accelerating the gas instead of ionizing it, which translates to higher thrust efficiency.

While MPDs utilize this plasma to build thrust, Hall-effect thrusters bombard neutral particles with electrons to build thrust. The electrons charge the propellant, which interacts with a combining electric and magnetic field to produce thrust. Multitudes of studies have gone into measuring the thrust efficiency - the energy output measured to energy input - of these thrusters. Their efficiencies are quite high, at times reaching $70 \%$ depending on the propellant used along with a variety of other factors (Kurzyna et al., 2018; Yang et al., 2019). The most significant benefit of using these thrusters over liquid-fuel thrusters is the high specific impulse of the propellant. Specific impulse is a measure of how efficiently a mass is used to build thrust - the higher the impulse, the less propellant is needed to generate the same thrust. Impulse is also directly correlated to the maximum speed of the craft, so a higher impulse means a potentially much quicker rocket. For electric engines, this high impulse is due to the immense exhaust speed in comparison to liquid-fuel rockets. While liquid rockets expel propellant at around 3 kilometers per second, propellant from a Hall-effect thruster can reach upwards of 80 kilometers per second. This is even higher in MPD thrusters, with speeds reaching 110 kilometers per second. The propulsion system with the highest impulse, however, is the photon thruster. Because photon thrusters use light as its propellant, its exhaust speed is the speed of light - approximately 300 thousand kilometers per second. Although the technology is in its beginning experimental phase, never having been implemented in rockets, recent data shows promising results. The potential for using photon thrusters for propulsion is huge, with estimates suggesting relativistic speeds for spacecraft being met with ease (Lubin, 2016).

While all the above engines expel propellant to create thrust, solar sails reflect it. Like sails on a boat, solar sails use the "wind" of photons coming from the sun to create thrust. The advantages of doing so are very significant. In addition to there being no on-board fuel required, it has been shown that solar sails are able to produce up to 9 newtons of force per square kilometer - a very sizable amount for a lightweight satellite (Macdonald \& McInnes, 2011). Electrodynamic tethers, like solar sails, require no physical fuel. These long cables, which can reach kilometers 
in length, are unique in that they can either produce thrust or build electrical energy depending on the situation. A celestial body's magnetic field interacts with the tether. If a converging magnetic field is created by sending a current through the tether, thrust is produced. However, if the tether moves across the body's field in a particular fashion, a magnetic flux is induced, generating electrical energy and decreasing height. In a peer-reviewed study, Alexandru Ionel of Romania's National Institute of Aerospace Research showed that tethers between 10 and 20 kilometers in length are able to produce up to a megawatt of electricity. Tethers of the same length can produce up to 200 newtons of force. A more recent innovation in electric propulsion that has the potential to revolutionize spaceflight is the airbreathing electric thruster. This engine is unique in that it allows there to be no on-board fuel in atmospheric conditions, using the surrounding air as fuel instead. As the rocket moves upward, air is led into an intake in a similar fashion to a jet engine. From there, the usual structural components of an electric thruster are put into place and the air is launched out of the craft at high speeds, producing thrust. The European Space Agency successfully fired the very first air-breathing electric thruster in 2018, citing long-lasting low earth orbit expeditions as the project's goal (European Space Agency, 2018). Successful advancement and application of air-breathing technology not only has the power to keep satellites in orbit for a longer time. Because atmospheric drag is greatly decreased due to the redirection of air into the thruster as fuel, satellites would be able to maintain a stable orbit at smaller heights.

However, the crucial question remains: with our current knowledge of physics and engineering, how can electric thrusters be best implemented to increase the efficiency of multi-stage rockets? Because of the new introduction of air-breathing thrusters onto the scene, this study primarily explores the possibility of using an air-breathing MPD in the first stage and other atmospheric stages of craft, implementing a quasi-experimental method to build data. The results of this experiment are then compared to peer-reviewed results of other forms of electric propulsion. This would not just show the most effective application of each type of thruster, but would also show how viable airbreathing MPDs currently are. An effective implementation of the technology, as well as that of other electric thrusters, has the potential to increase launch efficiency to where the payload's mass could be increased substantially while decreasing the net weight of the craft. The concept of air-breathing thrusters does not come without limitations, however. Because air is filled with diatomic molecules such as $\mathrm{O} 2$ or N2, the breakdown voltage is much higher. Therefore, more energy is devoted to ionization and less to building thrust, decreasing thrust efficiency. Paschen's law, which states that breakdown voltage is correlated with the product of distance and pressure, would also mean that breakdown requires a greater amount of energy near the surface (Paschen, 1889). Additionally, while propellant pressure can be fully controlled in the vacuum of space, the presence of an atmosphere creates a complicating factor. Among other limitations, there is that of sheer electrical power. Taking all of these into account, it is hypothesized that while airbreathing technology could be scaled to be a first-stage engine, comparative efficiency would limit the application of electric engines to the upper stages of rockets. Furthermore, it is hypothesized that electric engines in upper stages of crafts could be used in a more effective manner, especially when considering the possibilities of solar sails and electrodynamic tethers.

\section{Design and Experiment}

This study's quasi-experiment focuses on thrust efficiency instead of overall thrust. The resulting data could then be extrapolated to limits outside the reach of this study, giving way to a mathematical representation of a much stronger engine. Although this representation may not be perfect due to non-linearities measured in experimentation, physical and engineering relationships can be applied with significant precision. Maecker's Relation (Zhang et al., 2019)

$T$ represents the output force (thrust), $\mu$ represents the vacuum permeability constant, $J$ represents the electrical current, $r_{a}$ represents the radius of the anode, and $r_{c}$ represents the radius of the cathode.

$$
T=\frac{\mu J^{2}}{4 \pi}\left(\ln \frac{r_{a}}{r_{c}}+\frac{3}{4}\right)
$$


The first step in the experiment was to translate mathematical equations and standard physical models into a 2-dimensional representation of a working, breathing engine. Using the equation for the output force of a self-field MPD, also known as Maecker's Relation, it could be realized that a larger ratio between the anode and cathode radii increases thrust despite no increase in energy consumption. Thus, such a larger ratio increases thrust efficiency - the same amount of energy can be used to produce greater thrust. Although a maximization of the anode radius was attempted, limitations relating to cost kept it to 1.5 inches (3.81 centimeters) and the length to 6 inches (15.24 centimeters). The radius of the cathode, however, came to odds when considering the engine intake.

The specific shape of the intake was a subject of discussion in Stephen Jackson's master's thesis. Analyzations of tetrahedral, conical, and parabolic intakes consistently showed two relationships: percent capture increases as intake length does also, and parabolic intakes perform much better than the others in terms of specular reflection. Specular reflection is the "bouncing" of particles off a surface based on the angle of the surface and that of the particle's entry. A maximization of percent capture, making specular reflection close to $100 \%$, was also analyzed by Jackson. Minimizing the dead zones - the areas in the intake that do not reflect air into the ionization chamber - involved a process of finding the point of focus of the parabola. The focus entails a horizontal plane, called the directrix, below the vertex along with a central focal point above the parabola. Imaginary vertical lines connect the directrix and parabola, forming an intersection point. In this experiment, the anode also acts as the intake, simplifying the design of the engine to directly connect the intake and ionization chamber. Although this results in added weight in comparison to having the intake and chamber be separate, the pros of relative simplicity outweigh the cons in this experiment. For those that intend to analyze air-breathing thrusters as they are used in flight, separation of the two components is suggested. The cross-section of the cylindrical anode serves as the directrix and imaginary vertical lines connecting it to the parabola. If the vertical lines connect above the focal point (greater y-axis value), all points on the parabola achieve specular reflection. This would place reflection efficiency at $100 \%$ in theoretical, non-real systems. This number is near but not $100 \%$ for real systems due to imperfections in the parabolic curve. No matter what methodology is used, no intake could be a truly smooth parabola. The rounded equation $4.58 x^{2}-0.14$ was found when applying the limitations of a 4-inch length, leaving 2 inches for the ionization chamber.

Although breakdown voltage is linear at high pressure and distance, there is a nonlinear relationship at low values. Because less energy is used for ionization the smaller the gap is, thrust efficiency is inversely proportional to

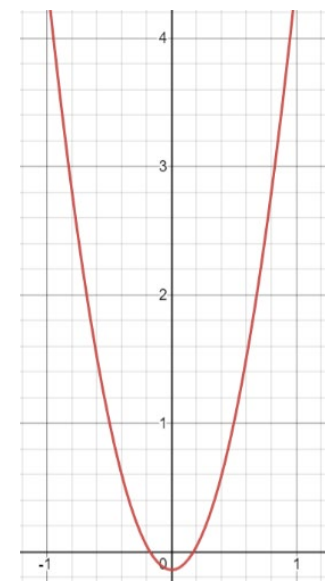

Figure 2. Graph of $4.58 x^{2}-0.14$

gap distance. Efforts therefore had to be made to make the gap between the anode and cathode as small as possible. Taking into account the intake, the space between the anode walls was calculated to be 0.25 inches in diameter (6.35 millimeters). A 6-millimeter diameter cathode rod was therefore built into the design, leaving gap distance to be 0.175 millimeters. 


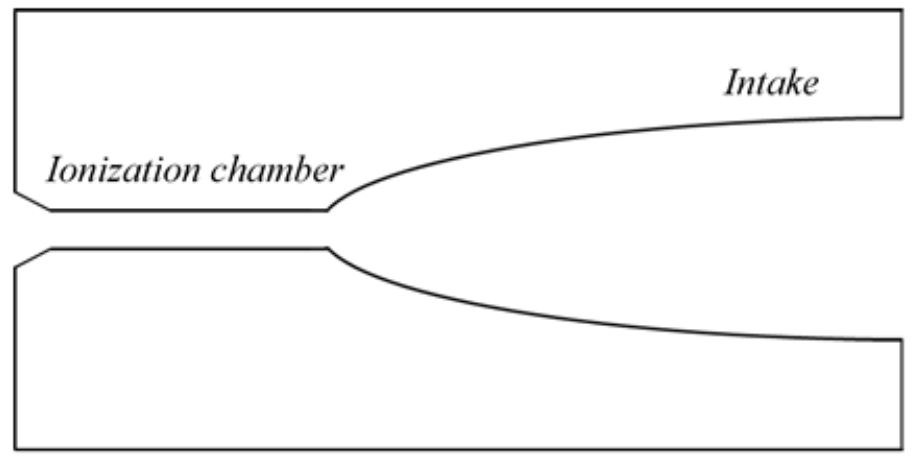

Figure 3. Designed MPD engine diagram, to scale

In order to achieve the engine's high voltage requirement, a boost converter was designed. The net effect of the converter is similar to a step-up electrical transformer - the voltage rises at the expense of current, maintaining conservation of power. The main difference between the two, however, is its application. Traditional transformers can only be applied to alternating current (AC), whereas converters are applied to direct current (DC). As my design implements the use of DC instead of AC, a boost converter is used for simplicity. A converter's design is split into 4 distinct parts - the power source, inductor, switch, and capacitor. A transistor is implemented into the design as the converter's switch due to its high frequency capabilities. Generally speaking, boost converters require a constant switching frequency of tens to hundreds of kilohertz - a speed of less than a millisecond per switch. Looking back at the MPD thrust equation, the square of the electrical current $J$ is proportional to trust. Thus, a decrease in current resulting from a boost converter decreases overall thrust. While this is a sacrifice to be made in this experiment, alternative solutions should be researched when attempting to maximize thrust instead of thrust efficiency.

After designing a 2-dimensional model of the engine and circuit surrounding it, the 3-dimensional engine in its entirety was built. It was clear that the engine's anode had to be made of copper due to the material's strong conductivity. As for the cathode, tungsten steel was selected due to its extremely high resistance to heat and high strength. Thus, a 3-inch diameter copper rod and 6-millimeter diameter tungsten rod were purchased. The engine intake involved a multi-step process. A series of drill holes were made in the copper rod, forming the shape of an uneven stairway that corresponds with the parabolic intake. A drill bit of a 1-inch diameter was first used, cutting into 3 inches of the copper. Next, 0.75-inch and 0.5-inch drill bits were used, digging an additional 0.6 and 0.275 inches respectively. Afterwards, thin copper sheets, laden with adhesive, were placed along the stairway to form a smooth

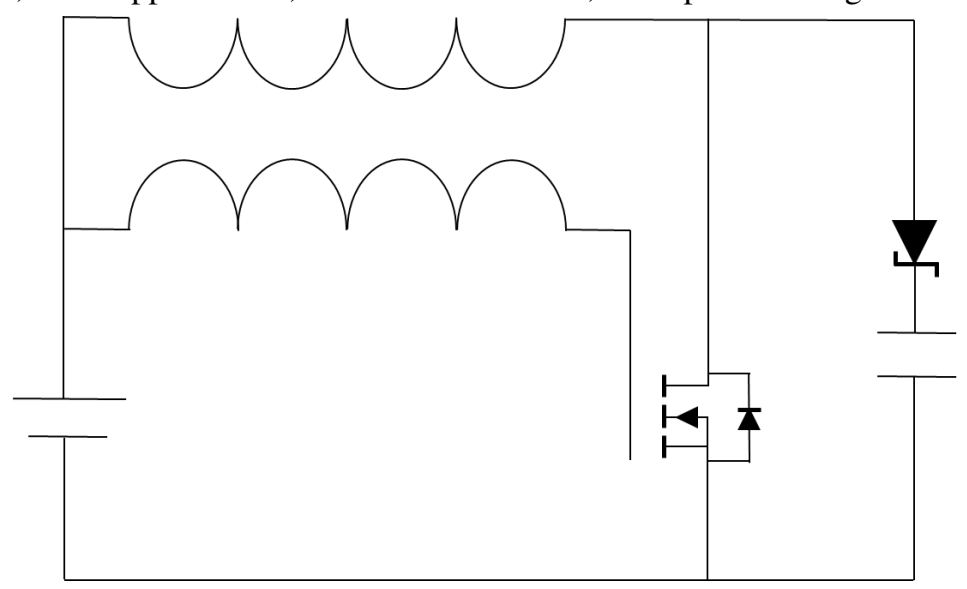

Figure 4. Designed boost converter circuit diagram 
curve. As the intake was being formed, an additional 0.25 -inch drill bit was used to create the engine's ionization chamber.

The boost converter circuit was built with a variety of converging variables in mind. First and foremost, it had to be designed to reach breakdown voltage without too many volts being inputted. A variable power source was also needed to establish relationships relating to thrust efficiency, so a series of up to ten 9-volt batteries was implemented. The inductor, a spiral of wiring that generates a magnetic field, must be able to handle the substantial initial current produced by the batteries. The American Wire Gauge scale, which is used for standardizing wire capabilities, was analyzed and used. 12 AWG, which can handle upwards of 1000 amperes at one-second pulses, was chosen. This wiring was then split into two parallel paths, as suggested by the 2-dimensional diagram (Figure 4). The first path connected the transistor to the inductor, with the gate pin connected to a separate feedback loop for switching operations. The transistor of choice was a MOSFET, part number IXFB110N60P3. MOSFETs, or Metal-Oxide-Semiconductor Field-Effect Transistors, are incredibly useful in boost converters due to their extremely high frequency switching capabilities. Most can reach hundreds of kilohertz - exponentially more than IGBTs, which are another highpower transistor type. The specific MOSFET used in this experiment was implemented because of its high voltage and current ratings, as well as its especially low switching time as per its datasheet. The second path of the boost converter connected the inductor to a Schottky diode and capacitor. Diodes are used in boost converters to prevent current from flowing from the second path back to the transistor in the first path. Specifically, Schottky diodes are used in the circuit because of their similar benefits to MOSFETs - the low amount of charge carried by the Schottky diode allows for exponentially greater switching frequencies relative to alternatives. With Schottky diodes, there is also an added benefit of a smaller voltage drop compared to alternatives. The diode of choice for this study was part number MSC050SDA070B, which was selected because of its substantial voltage and current ratings. The capacitor of choice was part number DCP5P06100D200KS00, a film capacitor selected for the same reasons.

After much trial and error, the purchased parts were connected, a multimeter also connected across the pins of the capacitor. Upwards of 600 volts were reached within mere seconds from a single 9-volt battery. Therefore, it would be reasonable to conclude that breakdown voltage needed to make the engine functional - around 1200 volts could be reached with an additional 9-volt battery. However, this idea was never brought into fruition due to a storm of newly understood safety issues. As the boost converter was tested, it was realized that the multimeter used had a maximum voltage rating of around 650 volts. After which, there would be no knowledge of how much energy was in the system. This would be incredibly dangerous due to capacitors' tendency to explode in dramatic fashion if too much energy was sent through them. If the circuit were to be connected for longer than it should have, there would be a considerable risk of explosion. An added safety concern was the discharging of the capacitor during testing. The small arcs produced from the high voltage combined with the somewhat significant current has the potential to be lethal. Taking this along with the already large time and financial constraints into account, this study had to be very quickly reshaped. Instead of producing real results through directly testing the built engine, which would have presented large aspects of danger, peer-reviewed models and mathematical equations were used to estimate what the results would have been. It must be noted that safety was the only concern in the development of results. The methodology and design of this study agrees completely with the review of academic literature present and are therefore valid for reproduction in safe environments.

\section{Results}

Maecker's Relation - the equation showing the output force of an MPD - can be applied to the built engine by considering the physical attributes of it. The radii of the anode and cathode of the thruster, which are 35.1 millimeters and 3 millimeters respectively, can be inputted into the equation to obtain a direct relationship between thrust and electrical current. 


$$
\begin{gathered}
T=\frac{\mu J^{2}}{4 \pi}\left(\ln \frac{r_{a}}{r_{c}}+\frac{3}{4}\right) \\
T=\frac{\mu J^{2}}{4 \pi}\left(\ln \frac{\mathbf{3 5 . 1}}{3}+\frac{3}{4}\right) \\
T \approx \frac{\mu J^{2}}{4 \pi}(3.2096) \\
T \approx 3.2096 \times 10^{-7} \times J^{2}
\end{gathered}
$$

Maecker's relation gives a rough estimation of thrust produced, but slight overestimations in the model were observed in academic literature. A 2012 study by Chu and his colleagues had an overestimation from a simulated model of around 12.5\%. In two other studies, one led by Tauchi in 2018 and another led by Zhang in 2019, overestimation from Maecker's relation was inversely proportional to thrust. However, it hovered around $10 \%$ and $20 \%$ respectively. The three overestimations were averaged, but more weight was placed on Tauchi and Zhang's studies due to greater similarities with the thruster made in this experiment. Chu's study revolved around an applied-field MPD, whereas the others implemented a self-field MPD, which was built in this study. This leads to the equation $T \approx 2.7282 \times 10^{-7} \times J^{2}$, a modified Maecker's relation that accounts for the approximated amount of overestimation that would have been present in this study's MPD. Graphing this curve shows impressive results.

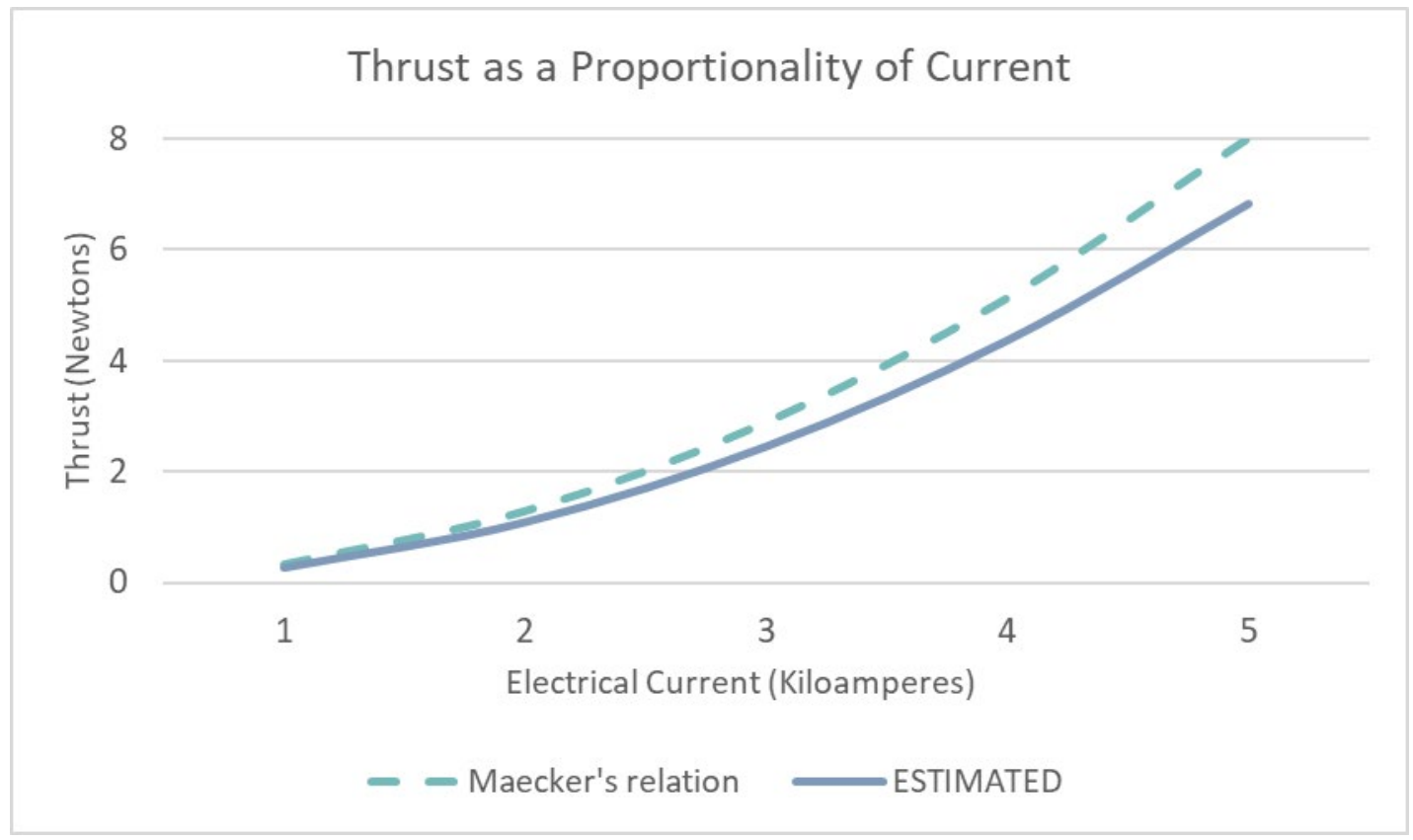

Figure 5. A graphical depiction of Maecker's relation and that accounting for overestimation 
In Figure 5, shown above, the dashed line represents an unmodified Maecker's relation applied to the MPD. The solid line, however, uses the equation found by taking into account overestimation. The thruster built would have had a base current of around 0.1 amperes due to the boost converter decreasing current to raise voltage, which translates to around 2.7 nanonewtons of thrust. However, because Maecker's relation can be easily extrapolated, data for a much greater current was graphed. This also results in a direct comparison between the built thruster and MPDs in peer-reviewed studies, due to there being larger amounts of power used in the studies. The thruster built in this experiment is estimated to have been $\sim 36 \%$ more current efficient than that built in Zhang's study, largely due to a greater ratio between the anode and cathode radii. This increase in efficiency is even higher when compared to a 2004 NASA study, written by researchers LaPointe, Strzempkowski, and Pencil. In comparison to the MPD built by these researchers, the MPD built in this study is estimated to have been $\sim 51 \%$ more current efficient. The graph below (Figure 6 ) shows thrust's relationship with electrical current for the three thrusters discussed.

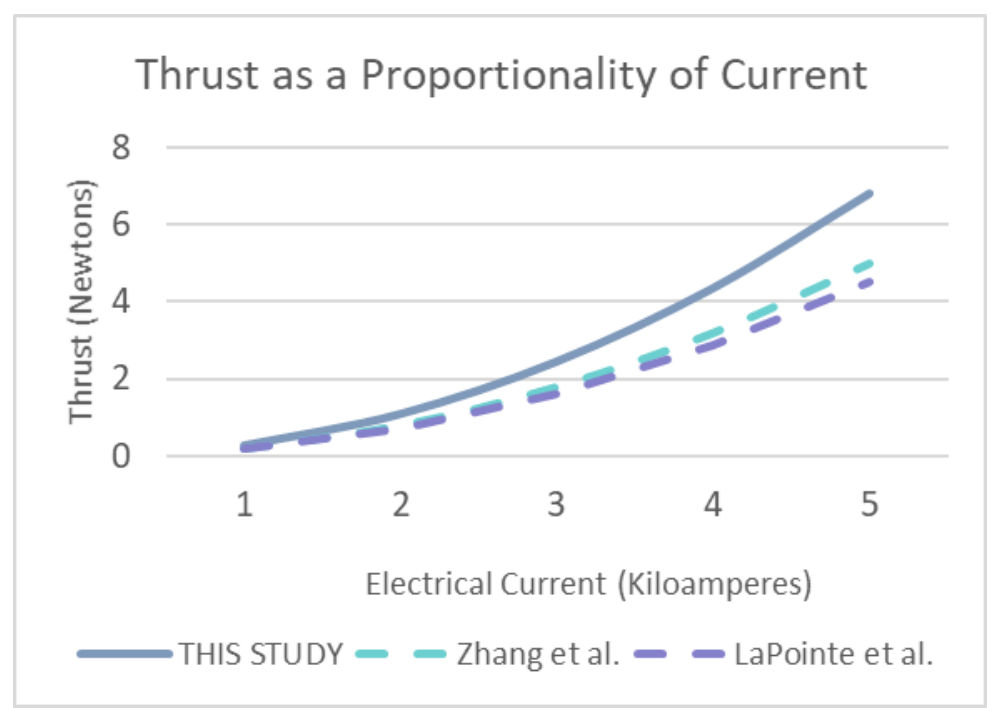

Figure 6. Thrust vs current between MPDs 
Although power efficiency was greatly increased, the high breakdown voltage of air greatly limits the overall thrust of the engine. Because much more energy is dedicated to turning air into plasma than other propellants, such as argon or krypton, power efficiency for air-breathing MPDs tends to be significantly lower than other thrusters. An effective way to measure power efficiency is to compare an engine's mass-to-thrust ratio with its input power. When the mass-to-thrust ratio is greater than or equal to one, meaning that thrust is equal to or larger than mass, a thruster is able to lift itself off the ground. This is a key, measurable factor that takes into account both increases in thrust efficiency and decreases in mass, which are both crucial components of rocketry. The higher the ratio is in comparison to its input power, the more efficient the thruster. The graph below depicts this relationship, using the combined mass of the anode and cathode of the built MPD - 59.16 Newtons - in doing so.

As shown by Figure 7, the built MPD thruster does not reach a mass-to-thrust ratio of 1 until approximately 17.6 megawatts are in the system. For scale, this is the equivalent of nearly 90 acres of solar panels. This figure does not account for the weight of the power storage system, which would only exponentially increase the energy required. In comparing the built MPD to a variety of other types of thrusters, the ideal type for specific missions is placed into consideration. Additionally, doing so can paint a picture of the development of air-breathing technology as of late, as well as how that development compares to similar developments in other systems. Air-breathing Hall-effect thrusters, solar sails, electrodynamic tethers, and photon thrusters are considered due to their relative popularity in spaceflight. The graphs below, figures 8 and 9, show power efficiency of the built MPD compared to different types of electric propulsion systems. To accurately compare the systems, helium replaces air as propellant in comparing the MPD to

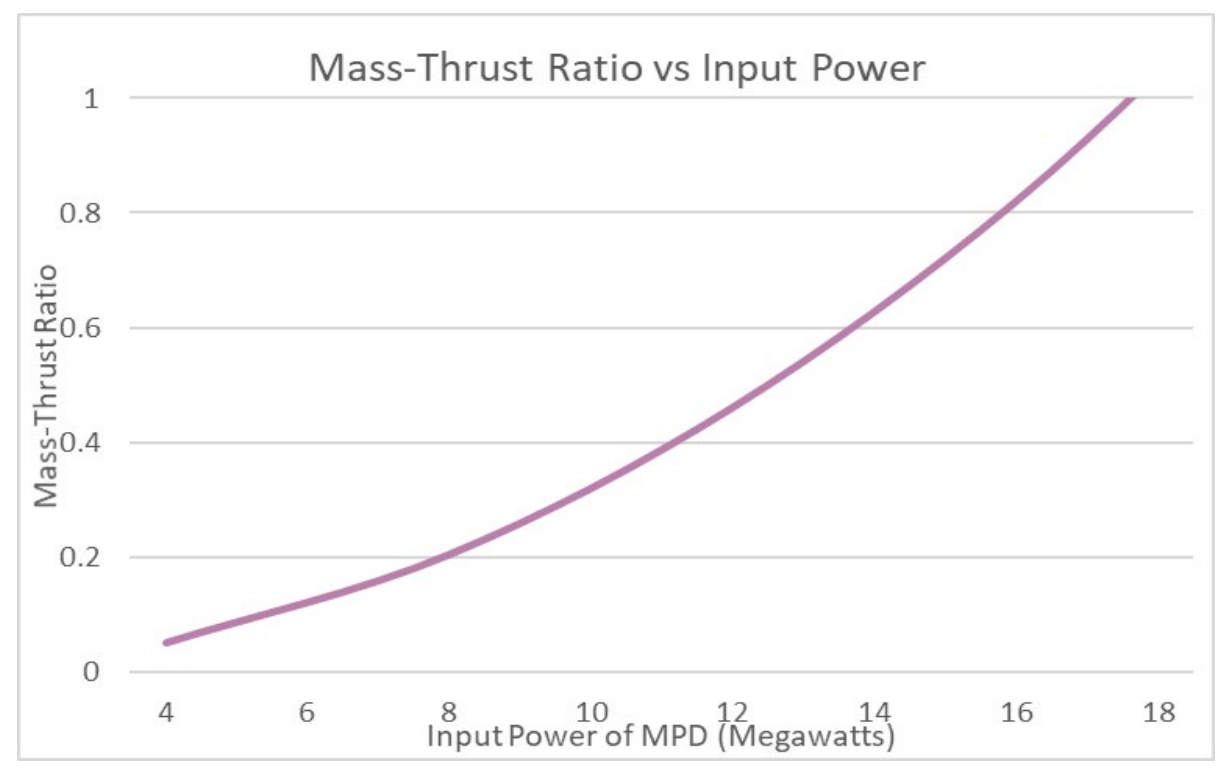

Figure 7. Graph showing the estimated mass-thrust ratio vs input power of the built MPD

electrodynamic tethers and solar sails. Helium was selected due to its extremely low breakdown voltage, which allows significantly more energy to be concentrated on producing thrust instead of breaking down the gas. While air-breathing technology cannot be directly compared to that of tethers and sails, a comparison between the propulsion systems and MPDs is nevertheless important. 
a.

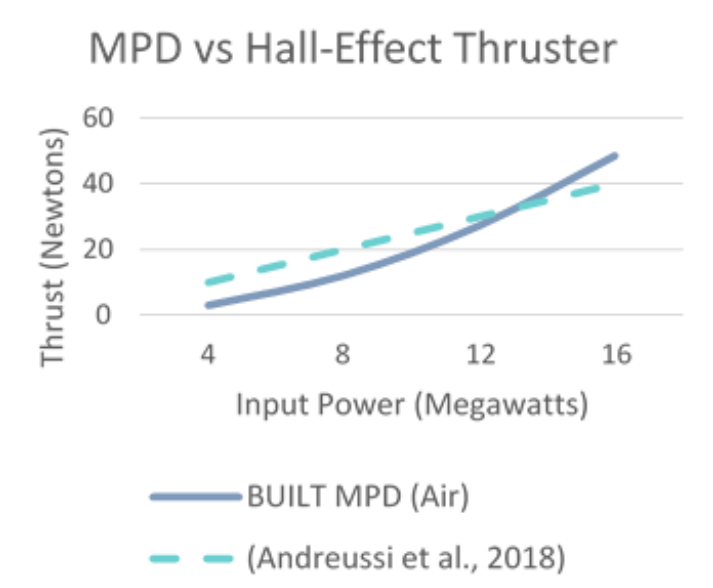

b.

\section{MPD vs Photon Thruster}

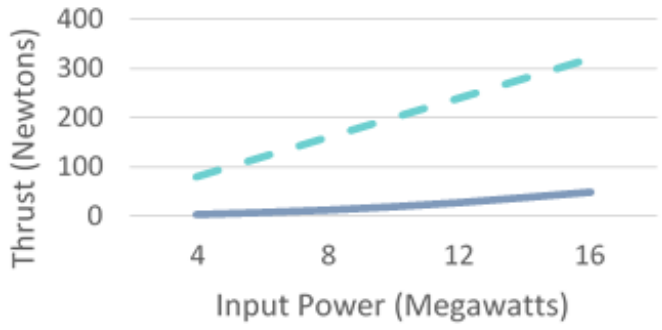

$\longrightarrow$ BUILT MPD (Air)

- (Bae, 2008)

Figure 8. The built MPD compared to an air-breathing Hall-effect thruster (a.) and photon thruster (b.)

a.

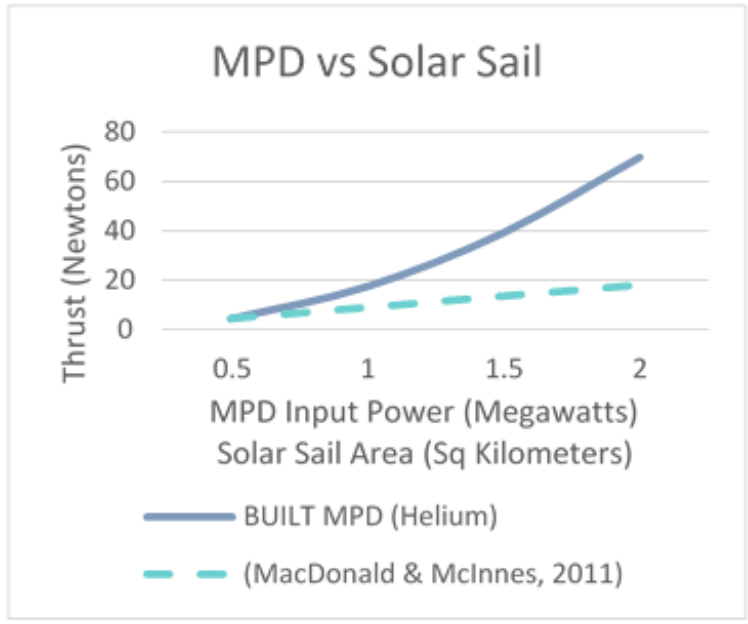

b.

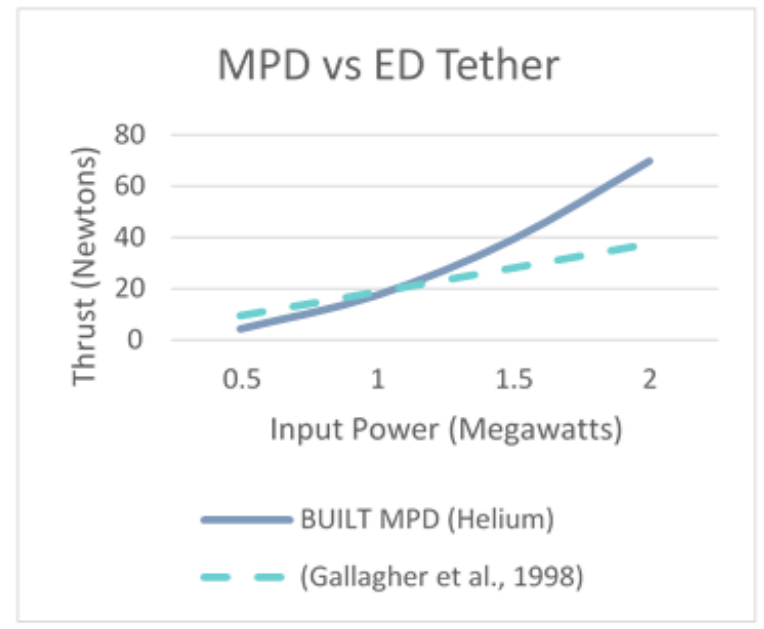

Figure 9. The built MPD with helium propellant compared to a solar sail (a.) and electrodynamic tether (b.)

As shown by the above graphs, comparable propulsion systems seem to produce more thrust per unit power than the built MPD up until a certain intersection point. This includes solar sails, which do not require any propellant or power to function. Kilometer-class solar sails would not only weigh exponentially less, and therefore have less inertia than a conventional electric thruster, they would also generate a large amount of thrust. This decreased weight through not holding any propellant and through the system itself weighing less reduces the needed thrust to complete a certain space-fairing mission. Therefore, while MPDs and other systems may be able to produce more thrust at high power, using sails could still result in a much larger benefit. Using electrodynamic tethers also seems to be more beneficial than conventional electric thrusters. In addition to weighing less, figure $9 \mathrm{~b}$ shows that tethers produce more thrust per unit power than helium-based MPDs up until about a megawatt.

In atmospheric conditions, photon thrusters out-perform both air-breathing MPDs and air-breathing Hall-effect thrusters with great significance. Bae's study was selected as a representative sample of photon thrusters due to his research 
organization's heavy focus on the technology. This focus leads to results that overshadow those of other studies. While Bae's photon thruster outpaces air-breathing technologies, conventional helium-based MPDs perform better at high power.

\section{A New Understanding}

While the quasi-results from this experiment yield efficiency increases in air-breathing MPDs, a comparison between it and other propulsion systems shows that the technology is currently not viable. Photon thrusters carry the same benefit as air-breathing thrusters in that no propellant is needed. However, thrust produced is greatly increased in comparison (Figure 8b) and its application extends beyond atmospheric conditions. There is also an added benefit of relative simplicity, as air-breathing thrusters rely on the ever-changing and not $100 \%$ predictable composition of the atmosphere. Photon thrusters therefore make for an ideal thruster type for low-earth orbit satellites if longevity of use is an important goal. However, if longevity is not an important goal, helium-based electric propulsion should be of use in atmospheric conditions. Moving beyond low-earth orbit, thruster types largely depend on the mission and should be reviewed on a case-by-case basis. However, as a general rule, passive electric propulsion such as electrodynamic tethers and solar sails can be immensely beneficial at lower power levels. Beyond a megawatt of input power, the exponentially increasing strength of MPDs overtakes that of tethers (Figure 9b). A long-proposed idea of how to achieve this level of power is an onboard nuclear fission reactor. Not only do fission reactors provide large amounts of electrical energy in lightweight masses, the products of the reaction could theoretically be used as propellant. This proposal has never been officially tested, but development of the idea is highly encouraged.

One of the most significant limitations of air-breathing MPDs is the large breakdown voltage of air. The concentration of energy on breaking down the propellant limits the amount of thrust the engine is able to produce; therefore, a lower breakdown voltage greatly increases thrust relative to power. To achieve this lower breakdown voltage, the electromechanics of breakdown should be further researched. It is known that breakdown decreases as relative humidity increases (Koch et al., 2007), so methodologies focusing on humidity's effects on MPDs should be explored. In addition to these variables, efficiency increases in photon thrusters should be further researched. Bae's study shows that even a slight increase in mirror reflectance has a stark increase in thrust efficiency. A maximization of reflectance as well as a minimization of laser input power has the potential to make photon thrusters increasingly mainstream in rocketry. Implementing these technologies at their fullest potential could revolutionize spaceflight forever, launching humanity into a new era of exploration.

\section{Acknowledgments}

I would like to thank my advisor Mr. John Murnan for helping me with this project.

\section{References}

Andreussi et al. (2018). Development and experimental validation of a Hall effect thruster RAM-EP concept. Space Propulsion 2018, Seville, Spain. https://www.researchgate.net/publication/337228639_DEVELOPMENT_AND_EXPERIMENTAL_VALI DATION_OF_A_HALL_EFFECT_THRUSTER_RAM-EP_CONCEPT

Bae, Y. K. (2008). Photonic laser propulsion: Proof-of-Concept demonstration. Journal of Spacecraft and Rockets, 45(1), 153-155. https://doi.org/10.2514/1.32284

Chu, K.-Y., Kubota, K., Funaki, I., \& Okuno, Y. (2012). Numerical investigations of a repetitively pulsed MPD thruster. IEEJ Transactions on Electrical and Electronic Engineering, 7(3), 234-239.

https://doi.org/10.1002/tee.21723 
Gallagher, D. L., Johnson, L., Moore, J., \& Bagenal, F. (1998, January). Electrodynamic tether propulsion and power generation at Jupiter. National Aeronautics and Space Administration. https://ntrs.nasa.gov/api/citations/19980203952/downloads/19980203952.pdf

Ionel, A. (2014). Numerical study of rocket upper stage deorbiting using passive electrodynamic tether drag. INCAS Bulletin, 6(4). https://doi.org/10.13111/2066-8201.2014.6.4.5

Jackson, S. W., \& Marshall, R. (2018). Conceptual design of an air-breathing electric thruster for CubeSat applications. Journal of Spacecraft and Rockets, 55(3). https://doi.org/10.2514/1.A33993

Koch, M., Fischer, M., Tenbohlen, S. (2007). The breakdown voltage of insulation oil under the influence of humidity, acidity, particles and pressure. Retrieved from https://www.researchgate.net/publication/306127998_The_breakdown_voltage_of_insulation_oil_under_th e_influence_of_humidity_acidity_particles_and_pressure.

Kurzyna, J., Jakubczak, M., Szelecka, A., \& Dannenmayer, K. (2018). Performance tests of IPPLM's krypton Hall thruster. Laser and Particle Beams, 36(1), 105-114. https://doi.org/10.1017/S0263034618000046

LaPointe, M., Strzempkowski, E., \& Pencil, E. (2004). High power MPD thruster performance measurements. American Institute of Aeronautics and Astronautics. https://doi.org/10.2514/6.2004-3467

Lubin, P. (2016, April 21). Photonic propulsion for relativistic flight: Enabling the first interstellar missions [video file]. Retrieved from https://iee.ucsb.edu/events/photonic-propulsion-relativistic-flight-enabling-firstinterstellar-missions.

Macdonald, M., \& McInnes, C. (2011). Solar sail science mission applications and advancement. Advances in Space Research, 48(11), 1702-1716. https://doi.org/10.1016/j.asr.2011.03.018

National Aeronautics and Space Administration. (2018, February 22). Space launch report: SpaceX falcon 9 v1.2 data sheet. https://sma.nasa.gov/LaunchVehicle/assets/spacex-falcon-9-v1.2-data-sheet.pdf

Paschen, F. (1889). Ueber die zum Funkenübergang in Luft, Wasserstoff und Kohlensäure bei verschiedenen Drucken erforderliche Potentialdifferenz [On the potential difference required for spark transfer in air, hydrogen and carbonic acid at different pressures]. Annalen der Physik. 273(5), 6975. https://doi.org/10.1002/andp.18892730505

Tauchi, S., Kawasaki, A., Nakane, M., Kubota, K., \& Funaki, I. (2018). The effect of anode configuration on hydrogen MPD thruster performance: A numerical study. Transactions of the Japan Society for Aeronautical and Space Sciences, Aerospace Technology Japan, 16(3), 274-279. https://doi.org/10.2322/tastj.16.274

World-first firing of air-breathing electric thruster. (2018, March 5). The European Space Agency. https:/www.esa.int/Enabling_Support/Space_Engineering_Technology/World-first_firing_of_airbreathing_electric_thruster

Yang, X., Hang, G., Cheng, M., Wang, M., \& Liang, W. (2019). Performance Evaluation of a 40-mN Hall thruster using laser-induced flourescence with comprehensive error analysis. IEEE Transactions on Plasma Science, 47(10), 4691-4699. https://doi.org/10.1109/TPS.2019.2937456

Zhang, Y., Wu, J., Ou, Y., Li, J., \& Tan, S. (2019). Modified electrodynamical modeling and parameters analysis of magnetoplasmadynamic thruster. Energies, 12(12). https://doi.org/10.3390/en12122428

Zheng, P., Wu, J., Zhang, Y., \& Wu, B. (2020). A comprehensive review of atmosphere-breathing electric propulsion systems. International Journal of Aerospace Engineering, 2020, 1-21. https://doi.org/10.1155/2020/8811847 